\title{
Determination of effective loss factors in reduced SEA models
}

\author{
M. Chimeno Manguán a,*, M.J. Fernández de las Heras ${ }^{b}$, E. Roibás Millán ${ }^{c}$, \\ F. Simón Hidalgo ${ }^{b}$
}

\begin{abstract}
A B S T R A C T
The definition of Statistical Energy Analysis (SEA) models for large complex structures is highly conditioned by the classification of the structure elements into a set of coupled subsystems and the subsequent determination of the loss factors representing both the internal damping and the coupling between subsystems. The accurate definition of the complete system can lead to excessively large models as the size and complexity increases. This fact can also rise practical issues for the experimental determination of the loss factors. This work presents a formulation of reduced SEA models for incomplete systems defined by a set of effective loss factors. This reduced SEA model provides a feasible number of subsystems for the application of the Power Injection Method (PIM). For structures of high complexity, their components accessibility can be restricted. for instance internal equipments or panels. For these cases the use of PIM to carry out an experimental SEA analysis is not possible. New methods are presented for this case in combination with the reduced SEA models. These methods allow defining some of the model loss factors that could not be obtained through PIM. The methods are validated with a numerical analysis case and they are also applied to an actual spacecraft structure with accessibility restrictions: a solar wing in folded configuration.
\end{abstract}

\section{Introduction}

The use of new materials and manufacturing processes has led to an increase of spacecraft structures size and complexity and also to a higher interest in their response at higher frequencies. One of the most widely used formulations for the high frequency range is Statistical Energy Analysis (SEA) [1]. SEA sets a system power balance as a function of a set of loss factors representing the internal damping and the coupling between subsystems (defined as a collection of natural modes with similar characteristics of each element of the system [1]). A fundamental assumption of the SEA theory is that an SEA mode] or system should include all the interacting elements that have to be defined in terms of SEA subsystems. This allows us to take into account all the possible power transmission paths involved. In this sense and, specially, for complex structures, the selection of the minimum number of elements that takes into account all relevant power flows is an important issue.

If all the elements that interact among them are not included in the analysis then the system considered is incomplete. For example, if the surrounding air or some interior inaccessible elements are not included. The response of such an 
incomplete system is ruled by a smaller model that may not define a proper power equilibrium. This case can be extended straightforwardly to the definition of smaller models in large and complex structures. Complex systems are usually composed of elements with a different level of interest from the point of view of the response. Elements of a system can be classified in: primary ones, if their response is of high interest; and secondary ones, if their response is of low interest from the engineering point of view. The development of models focused on the primary elements leads to smaller and easier to use models.

If only the primary elements are considered as subsystems, their internal loss factors (ILF) and coupling loss factor (CLF) should take into account the interaction with secondary elements if the power flow involved is significant. As it will be shown, this fact has influence in both the direct problem (the system response is calculated from the power input) and the inverse problem (determining the model loss factors from the system response). For systems with significant interaction between primary and secondary subsystems, a new formulation is presented. The formulation is based on the definition of a set of effective loss factors and a reduced SEA loss matrix that allows for this interaction to be defined.

For both complete and incomplete systems, the determination of the loss factors of an actual complex structure is the main and first issue to define an SEA model. For simple elements an analytical [1| or numerical approach [2] can be considered. However for an actual structure the feasible and standard approach is based on in situ measurements to determine the system power balance. The first proposal to determine experimentally the loss factors was the Power Injection Method (PIM) proposed by Bies and Hamid [3] followed by some alternative equations to deal with numerical problems [4,5]. PIM and all derived techniques lie in the measurement of the whole system power balance for different power inputs (as many as subsystems are considered).

This requirement may not be fulfilled for a complex structure because some elements have accessibility restrictions for exciting them or measuring their response [6,7]. If this is the case, a direct application of PIM is not possible and some alternative formulations would be needed. The Power Coefficient Method (PCM) [8] does not require the measurement of the input power but the measurement of the system response. This method allows us to define an alternative SEA model based on the power coefficients instead of loss factors. Another alternative is based on a transmissibility formulation [9] which does not require the measurement of the power input although it requires to know the internal or total loss factors of the subsystems being analysed. The present work describes alternative approaches to determine the coefficients of the SEA model for cases of partial excitation or partial measurement, whose analysis could not be addressed by PIM and techniques alike.

This paper is organised as follows. In Section 2 the formulation of SEA models for incomplete systems is presented. The usual approach is compared with a new formulation based on reduced SEA models. In Section 3 alternative methods are presented to determine the model matrix coefficients for cases in which not all the elements can be measured or excited. In Section 4 a numerical study case is presented to validate the formulation and methods presented. In Section 5 an application case consisting on an actual solar wing is analysed by means of the new methods. The conclusions derived from the work are summarised in Section 6.

\section{SEA models of incomplete systems}

Prior to the classification of the system sub-structures into a set of subsystems, the limits of the system itself have to be defined. For a system to be considered complete, it has to include all the elements that interact between them. In other case, the definition of the system is incomplete and some interaction is left out of the system. For a complete system of $n_{s}$ subsystems the SEA theory expresses the power balance within the system, in a frequency band centred in $\omega$, in terms of the power balance for each subsystem $i$ :

$$
P_{i}=\omega \eta_{i i} E_{i}+\omega \sum_{\substack{j=1 \\ j \neq i}}^{n_{s}}\left(\eta_{i j} E_{i}-\eta_{j i} E_{j}\right) \quad i=1: n_{s}
$$

where $P_{i}$ stands for the input power due to external loads, $E_{i}$ and $E_{j}$ are the energies of subsystems $i$ and $j$ respectively, $\eta_{i i}$ is the ILF of subsystem $i$ and $\eta_{i j}\left(\eta_{j i}\right)$ is the CLF from subsystem $i(j)$ towards subsystem $j(i)$.

The power balance of the system in a frequency band centred in $\omega$ can be expressed then as

$$
\mathbf{P}=\omega \mathbf{L E}
$$

where $\underline{\mathbf{P}}$ and $\underline{\mathbf{E}}$ are vectors composed of the $n_{s}$ input powers into each subsystem and their energies respectively, and $\mathbf{L}$ is the SEA loss matrix of the model, whose coefficients are defined from the loss factors as

$$
l_{i j}= \begin{cases}\sum_{k=1}^{n_{s}} n_{i k} & i=j \\ -\eta_{j i} & i \neq j\end{cases}
$$

Although the SEA formulation leads to significantly smaller models than those methods based on low frequency 
formulations as finite element models (FEM), the increase in size and complexity of the structures leads to relatively large SEA models. This fact leads to practical problems for defining and characterising the interaction between the different subsystems. Also, in large systems their elements can usually be classified in terms of the interest in their response and also in terms of their influence on the whole system performance (as primary and secondary elements). To develop a smaller model focused on these primary subsystems two different approaches can be considered that are presented in the following sections: first, the current usual procedure based on defining a smaller model erasing the equations and terms of the secondary subsystems [10] (assuming they can be neglected) and second, a new formulation to define a reduced model that takes into account explicitly the secondary elements.

\subsection{Truncated SEA models}

If the subsystems that make up a system are classified into primary and secondary subsystems, Eq. (2) can be partitioned into

$$
\left\{\begin{array}{l}
\underline{\mathbf{P}}_{A} \\
\underline{\mathbf{P}}_{O}
\end{array}\right\}=\omega\left[\begin{array}{ll}
\mathbf{L}_{A A} & \mathbf{L}_{A O} \\
\mathbf{L}_{O A} & \mathbf{L}_{O O}
\end{array}\right]\left\{\begin{array}{l}
\underline{\mathbf{E}}_{A} \\
\underline{\mathbf{E}}_{O}
\end{array}\right\}
$$

where subscripts $A$ and $O$ stand for the $n_{a}$ primary subsystems to be analysed and the $n_{o}$ secondary subsystems to be omitted.

The usual procedure to reduce the analysis model size is to use the corresponding submatrix $L_{A A}$

$$
\mathbf{P}_{A}=\mathbf{L}_{A A} \underline{\mathbf{E}}_{A}
$$

Therefore, the coefficients of the truncated model $\mathrm{L}_{A A}$ are

$$
l_{A A_{i j}}= \begin{cases}\sum_{i=1}^{n_{t}} \eta_{A_{j} A_{t}}+\sum_{i=1}^{n_{0}} \eta_{A_{i} O_{t}} & i=j \\ -\eta_{A_{j} A_{j}} & i \neq j\end{cases}
$$

Although the truncated model is focused on the primary (analysed) subsystems, Eq. (6) shows that the power flow from the analysed to the omitted subsystems is taken into account through the second term in the diagonal coefficients. Nevertheless it does not include the whole interaction between both types of subsystems as the off-diagonal entries in Eq. (6) include only the direct interaction between analysed subsystems. That is to say, the coupling between the primary and secondary subsystems is taken into account by an increase of the value of the total loss factors (TLF) that accounts for all the power getting into the secondary subsystems.

For those cases in which the power flow from analysed subsystems through omitted ones is negligible, the truncated model provides an adequate model of the system. By contrast, the truncated model $\mathbf{L}_{A A}$ may not reproduce adequately the system response if the contribution of the omitted subsystems through higher order paths is noticeable. In such cases, the interaction between analysed subsystems would be underestimated in the response calculation (direct problem), as it would not include the indirect contribution through omitted subsystems. If the system parameters are being calculated (inverse problem), the internal loss factors of the analysed subsystems would be overestimated.

\subsection{Reduced SEA models}

As stated, previous method can lead to reduce the accuracy of the model parameters and it does not retain explicitly the influence of the omitted subsystems. A new formulation based on a reduced SEA model is presented in this section to overcome these problems. A model reduction technique [11] is used to define a smaller SEA mode] for the balance between the input powers and the energies of the analysed subsystems.

The static reduction scheme formulated by Guyan [12] is used on the assumption that the input power to the secondary subsystems is null. This hypothesis is referred to the external loads only and it is feasible in large complex structures whose internal elements are only subjected to external loads. For example, internal elements in spacecraft structures that are not directly exposed to external acoustic loads; secondary structures in train wagons not supporting equipments or interior panels in ships.

If the input power to the $n_{o}$ omitted subsystems is null $\left(\underline{\mathbf{P}}_{0}=\mathbf{0}\right)$ their energy can be solved from the lower block of Eq. (4)

$$
\underline{\mathbf{E}_{O}}=-\mathbf{L}_{O}^{-1} \mathbf{L}_{O A} \mathbf{E}_{A}
$$

Introducing Eq. (7) into the upper block of Eq. (4) the power balance of the system reducing out the $n_{o}$ omitted subsystems is expressed as

$$
\underline{\mathbf{P}}_{A}=\omega \mathbf{L}^{\left(\boldsymbol{n}_{0}\right)} \underline{\mathbf{E}}_{A}
$$

where 


$$
\mathbf{L}^{\left(n_{0}\right)}=\mathbf{L}_{A A}-\mathbf{L}_{A O} \mathbf{L}_{O O}^{-1} \mathbf{L}_{O A}
$$

is the reduced SEA loss matrix of order $\left(n_{o}\right)$.

Unlike the truncated model, the reduced model retains explicitly the contribution of the omitted subsystems, since the coefficients of the reduced SEA loss matrix are based on the loss factors of both analysed and omitted subsystems. This formulation also provides the definition of the model coefficients for any selection of analysed and omitted subsystems, given that the omitted ones are not excited. Moreover, as the reduced SEA loss matrix is defined through operations on submatrices of an SEA loss matrix it might not satisfy the sign pattern of the SEA loss matrix coefficients.

The coefficients of the reduced SEA loss matrix can be defined through a recurrence relation starting from the SEA matrix of the complete system, i.e. reduced SEA loss matrix of order ( 0 ). If only one subsystem is reduced in each step $\mathbf{L}_{A O}$ and $\mathbf{L}_{O A}$ become column and row vectors respectively and $\mathbf{L}_{00}$ is the total loss factor of the omitted subsystem. Then in each reduction step Eq. (9) becomes an algebraic operation. For a general case of reducing $k$ subsystems, assuming that the subsystem to be reduced is placed on the last row/column of the SEA loss matrix, and for a system of $n_{5}$ subsystems of which $n_{a}$ are analysed and $n_{0}=n_{s}-n_{a}$ are omitted, the coefficients of the reduced SEA loss matrix of order $(k)$ are

$$
l_{i j}^{(k)}=l_{i j}^{(k-1)}-\frac{l_{i t}^{(k-1)} I_{i j}^{(k-1)}}{l_{i j}^{(k-1)}} \quad i_{i} j=1: n_{i} ; t=n_{a}+1 ; \text { and } k=1: n_{o}
$$

Second term in Eq. (10) shows that the contribution of the omitted subsystems is taken into account not only on the diagonal coefficients (as it was the case in the truncated model) but also in the off-diagonal elements. Therefore it takes into account the indirect interaction of analysed subsystems through the omitted ones.

By analogy with the relationship between the loss factors and the coefficients of the SEA loss matrix of a complete system, a set of effective loss factors (ELF) of order $(k)$ are defined. Both the effective internal loss factors (EILF) and the effective coupling loss factors (ECLF) of order $(k)$ are defined from the coefficients of the reduced loss matrix of order $(k)$ as

$$
f_{i j}^{(k)}= \begin{cases}\sum_{t=1}^{n_{s}-k} l_{i j}^{(k)} & i=j \\ -I_{j i}^{(k)} & i \neq j\end{cases}
$$

It follows that the effective loss factors of order (0), defined from the SEA loss matrix of the complete system, are directly related to the damping of the system.

\section{Experimental SEA of reduced SEA models}

Although the coefficients of a reduced SEA model can be defined through the usual approaches already stated, this section is focused in its experimental characterisation. Essentially, the different techniques proposed for this Experimental Statistical Energy Analysis (ESEA) [10] are based on the Power Injection Method described in [3]. For a set of $n_{a}$ analysed subsystems, the effective loss factors constitute a set of $n_{a}^{2}$ unknowns. Therefore $n_{a}$ different power balances are needed to define the $n_{a}^{2}$ equations required. The resulting set of $n_{a}^{2}$ equations can be formulated through matrix algebra as

$$
\mathbf{P}=\omega^{\left(\mathbf{n}_{0}\right)} \mathbf{E}
$$

where $\mathbf{P}$ is a matrix whose columns are the different sets of input powers and $\mathbf{E}$ is a matrix whose columns are the corresponding sets of energies of the analysed subsystems.

In this approach the response of all the analysed subsystems must be measured for $n_{a}$ different input power sets applied on all of them. For large and complex structures with interior structural elements, for instance, air layers, this requirement may not be fulfilled and therefore PIM cannot be applied to determine the whole set of loss factors. This section presents a new formulation to calculate the effective loss factors of a model from the input powers and energies of a structure for both partial excitation and partial measurement.

To this end, the set of $n_{a}$ analysed subsystems is classified into those whose response can be measured and can be excited, $n_{e}$, and those others that can be measured but cannot be excited, $n_{m}$. It follows straightforwardly that $n_{s} \geq n_{a} \geq\left\{n_{e}, n_{i n}\right\}$.

For those cases, which the PIM and techniques alike cannot be applied to, two alternative methods are suggested. First, a method based on minimising a cost function defined as the norm of the difference between the simulated and measured power matrices. Second, a method based on model updating techniques from a starting model subjected to the response measured.

\subsection{Complete measurement with partial excitation}

If all the $n_{a}$ analysed subsystems can be measured, $n_{m}=n_{a}$, and only a number of them can be excited (or their input power cannot be measured), $n_{e}<n_{a}$, then the input power and energy matrices $\mathbf{P}$ and $\mathbf{E}$ in Eq. (12) are not square any more 
but of size $n_{a} \times n_{e}$. Under these conditions an ordinary PJM cannot be used. Nevertheless, the two methods sketched below can be used to determine the reduced SEA loss matrix of order $n_{0}=n_{s}-n_{a}$ of size $n_{a} \times n_{a}$.

\subsubsection{Cost function minimisation method}

This method is based on obtaining the reduced SEA loss matrix that minimises the Euclidean norm defined by:

$$
J_{1}\left(\mathbf{L}^{\left(n_{S}-n_{\omega}\right)}\right)=\left\|\mathbf{P}-\omega \mathbf{L}^{\left(n_{s}-n_{0}\right)} \mathbf{E}\right\|
$$

This method may include constraints on the solution based on the system characteristics as for example symmetries, same ILF for identical elements, or null direct interaction between subsystems.

\subsubsection{Model updating method}

Depending on the system characteristics and the order of the reduced SEA loss matrix, convergence of $J_{1}$ may be slow or infeasible. On the other hand, the study of a structure does not usually start from the scratch but from previous developments that can be used as a prior information. For those cases a second method is proposed to improve a previous model

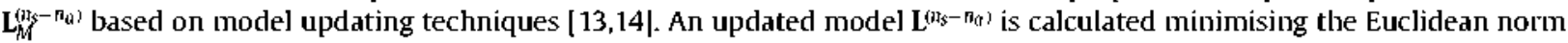

$$
J_{2}=\left\|\mathbf{L}^{\left(n_{s}-n_{Q}\right)}-\mathbf{L}_{M}^{\left(n_{s}-n_{a j}\right.}\right\|
$$

subject to the set of power balances measured

$$
\mathbf{P}=\omega \mathbf{L}^{\left(n_{s}-n_{a}\right)} \mathbf{E}
$$

The constraints given by Eq. (15) represent $n_{a} \times n_{e}$ equations in the elements of the updated model $\mathbf{L}^{\left(n_{S}-n_{a}\right)}$. The Lagrange multiplier method [15] can be used then to define an augmented function whose minimisation takes into account the power balance measured

$$
J_{2}=\sum_{i, j=1}^{n_{a}}\left[\left(\mathbf{L}^{\left(n_{s}-n_{a}\right)}\right)_{i j}-\left(\mathbf{L}_{M}^{\left(n_{s}-n_{(j)}\right)}\right)_{i j}\right]^{2}+\sum_{j=1}^{n_{a}} \sum_{j=1}^{n_{e}}(\lambda)_{i j}\left[(\mathbf{P})_{i j}-\omega \sum_{k=1}^{n_{a}}\left(\mathbf{L}^{\left(n_{s}-n_{a}\right)}\right)_{i k}(\mathbf{E})_{k j}\right]
$$

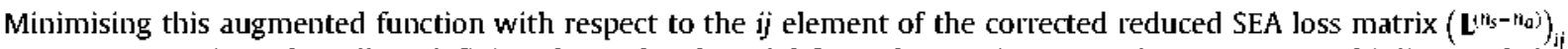
gives $n_{a} \times n_{e}$ equations that allow defining the updated model from the previous one, the Lagrange multipliers and the measured energy

$$
\mathbf{L}^{\left(n_{s}-n_{a j}\right)}=\mathbf{L}_{M}^{\left(n_{s}-n_{\omega}\right)}+\frac{1}{2} \omega \lambda \mathbf{E}^{\top}
$$

Introducing Eq. (17) into Eq. (15) allows obtaining the Lagrange multipliers

$$
\lambda=\frac{2}{\omega^{2}}\left(\mathbf{P}-\omega \mathbf{L}_{M}^{\left(\pi_{s}-n_{a}\right.} \mathbf{E}\right)\left(\mathbf{E}^{T} \mathbf{E}\right)^{-1}
$$

Introducing this result into Eq. (17) determines the updated reduced SEA loss matrix

$$
\mathbf{L}^{\left(n_{s}-n_{a}\right)}=\mathbf{L}_{M}^{\left(n_{S}-n_{\omega}\right)}+\frac{1}{\omega}\left(\mathbf{P}-\omega \mathbf{L}_{M}^{\left(m_{s}-n w_{0}\right)} \mathbf{E}\right)\left(\mathbf{E}^{T} \mathbf{E}\right)^{-1} \mathbf{E}^{\top}
$$

Therefore, if all the analysed subsystems are measured in the power balance test (complete measurement), but not all of them are excited (partial excitation) this analytical updating rule can be used to improve the initial model from the experimental results.

\subsection{Partial measurement}

If not all the $n_{a}$ analysed subsystems can be measured, $n_{n}<n_{b}$, the measured response is determined by the reduced SEA loss matrix of order $\left(n_{a}-n_{m}\right)$ of size $n_{m} \times n_{n}$, while the model defined for the $n_{a}$ subsystems is larger of size $n_{a} \times n_{a}$. The methods proposed still apply to this case although some considerations have to be taken into account in the definition of both $J_{1}$ and $J_{2}$.

The cost function of the minimisation method depends explicitly on the reduced SEA loss matrix of order ( $\left.n_{s}-n_{m}\right)$ in the form of

$$
J_{1}\left(\mathbf{L}^{\left(n_{s}-n_{m}\right)}\right)=\left\|\mathbf{P}-\omega \mathbf{L}^{\left(n_{s}-n_{m}\right.} \mathbf{E}\right\|
$$

Moreover, the second method takes into account both the model to be updated (of size $n_{a} \times n_{a}$ ) and the matrix related to the power balance of the system measured experimentally (of size $n_{n} \times n_{n}$ ). For the case of partial measurement the updated model $\mathbf{L}^{\left(t_{s}-n_{a} \text { ! }\right.}$ is calculated by minimising the norm in Eq. (14) subjected to the set of power balances measured

$$
\mathbf{P}=\omega \mathbf{L}^{\left(r_{S}-\pi m\right)} \mathbf{E}
$$


In both cases, matrices $\mathbf{P}$ and $\mathbf{E}$ are composed of the measured power balances. Both have $n_{m}$ rows corresponding to the number of subsystems that can be measured and the number of columns depends on the excitation case: (a) for complete excitation (all measured subsystems can be excited) they have $n_{e}=n_{m}$ columns being then square matrices, (b) for partial excitation they have $n_{e}<n_{m}$ columns assuming that if the subsystem can be excited then it can be measured.

\subsection{Implementation}

Except for the case covered by the updating law in Eq. (19), which requires to measure all the subsystems analysed and a previous model to be updated, a numerical implementation is required.

Within this work a multi-start heuristic algorithm [16] is used to minimise $J_{1}$ in Eqs. (13) and (20) and $J_{2}$ in Eq. (14) subject to Eqs. (15) or (21). The algorithm is based on an initial scatter search algorithm [17] combined with an analysis of the efficiency of each design space point to provide the global minimum in the following step. Next, a set of gradient based NLP (nonlinear programming) solvers is used starting with the selected points until the global minimum of the solution space is found.

\section{Validation case}

A reference case is presented to validate the formulation of the reduced SEA loss matrix and the methods used to calculate the effective loss factors. The case consists of three parallel identical flat panels separated from each other by air cavities. The panels size is $1.0 \times 1.25 \times 0.005 \mathrm{~m}$ and they are made up of aluminium with density $2750 \mathrm{~kg} / \mathrm{m}^{3}$. The air layers are $50 \mathrm{~mm}$ thick. A density of $1.225 \mathrm{~kg} / \mathrm{m}^{3}$ and a sound speed of $343 \mathrm{~m} / \mathrm{s}$ are assumed.

The complete system is composed of five elements: each panel is modelled as a bending plate subsystem and each air layer as an acoustic cavity, an usual model for this type of system is adopted here [18,19]. Fig. 1 shows the blocks diagram of the interactions between SEA subsystems.

This SEA model loss factors - effective loss factors of order (0) - can be defined analytically. The loss factors can be defined as found in the literature from: the modal density of the panels [20] and the air cavity [21]; the radiation efficiency of a panel into an acoustic cavity $[22,23]$ and the reciprocity relationship between SEA subsystems $i$ and $j$, i.e. $n_{i} n_{i j}=n_{j} \eta_{j i}$ where $n_{i}$ and $n_{j}$ stand for the modal density of each subsystem. Non-resonant transmission through the panels [18] and through the air layers [19] are also included. The internal loss factor of the five subsystems is set to 0.01 .

Hereinafter $\eta_{s}$ and $\eta_{f}$ stand for the ILFs of the structural panels and the air layers respectively; $\eta_{s f}\left(\eta_{f s}\right)$ stands for the resonant transmission from a panel (air layer) to the contiguous air layers (panels); $\eta_{f f}$ stands for the CLF between air layers due to the non-resonant transmission through the inner panel and $\eta_{s s}$ is the CLF between panels due to the non-resonant transmission through the air layer between them. The SEA loss matrix of the system considering the panels as subsystems 1 , 3 and 5 , and the air layers as subsystems 2 and 4 is

$$
\mathbf{L}=\left[\begin{array}{ccccc}
\eta_{s}+\eta_{s f}+\eta_{s s} & -\eta_{f s} & -\eta_{s s} & 0 & 0 \\
-\eta_{s f} & \eta_{f}+2 \eta_{f s}+\eta_{f f} & -\eta_{s f} & -\eta_{f f} & 0 \\
-\eta_{s s} & -\eta_{f s} & \eta_{s}+2 \eta_{s f}+2 \eta_{s s} & -\eta_{f s} & -\eta_{s s} \\
0 & -\eta_{f f} & -\eta_{s f} & \eta_{f}+2 \eta_{f s}+\eta_{f f} & -\eta_{s f} \\
0 & 0 & -\eta_{s s} & -\eta_{f s} & \eta_{s}+\eta_{s f}+\eta_{s s}
\end{array}\right]
$$

Reduction according to Eq. (9) where the structural panels are the ones analysed leads to the reduced SEA loss matrix of order (2)

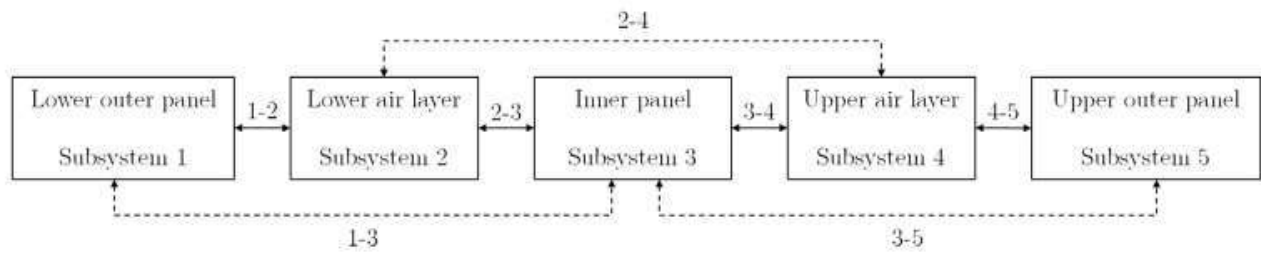

Fig. 1. The Statistical Energy Analysis model of the validation case. 


$$
\mathbf{L}^{(2)}=\left[\begin{array}{ccc}
\eta_{s}+\eta_{s f}+\eta_{s s}-\frac{\eta_{s f} \eta_{f s}\left(\eta_{f}+2 \eta_{f s}+\eta_{f f}\right)}{\left(\eta_{f}+2 \eta_{f s}\right)\left(\eta_{f}+2 \eta_{f s}+2 \eta_{f f}\right)} & -\eta_{s s}-\frac{\eta_{s f} \eta_{f s}}{\eta_{f}+2 \eta_{f s}} & -\frac{\eta_{f s} \eta_{s f} \eta_{f f}}{\left(\eta_{f}+2 \eta_{f s}\right)\left(\eta_{f}+2 \eta_{f s}+2 \eta_{f f}\right)} \\
-\eta_{s s}-\frac{\eta_{s f} \eta_{f s}}{\eta_{f f}+2 \eta_{f s}} & \eta_{s}+\eta_{s f}+\eta_{s s}-\frac{2 \eta_{s f} \eta_{f s}}{\eta_{f}+2 \eta_{f s}} & -\eta_{s s}-\frac{\eta_{s f} \eta_{f s}}{\eta_{f f}+2 \eta_{f s}} \\
-\frac{\eta_{s} \eta_{s f} \eta_{f f}}{\left(\eta_{f}+2 \eta_{f s}\right)\left(\eta_{f}+2 \eta_{f s}+2 \eta_{f f}\right)} & -\eta_{s s}-\frac{\eta_{s f} \eta_{f s}}{\eta_{f}+2 \eta_{f s}} & \eta_{s}+\eta_{s f}+\eta_{s s}-\frac{\eta_{s f} \eta_{f s}\left(\eta_{f}+2 \eta_{f s}+\eta_{f f}\right)}{\left(\eta_{f}+2 \eta_{f s}\right)\left(\eta_{f}+2 \eta_{f s}+2 \eta_{f f}\right)}
\end{array}\right]
$$

\subsection{Partial excitation}

The new methods proposed for the determination of the effective loss factors are intrinsically appropriate to study cases of partial excitation unlike the PIM, as described in Section 3. Now, the excitation on the middle panel is considered infeasible. Such is the case of interior elements of spacecraft structures or solar wings in folded configuration, for which access to the inner area of the middle panels is not possible. Nevertheless, the energy in all the panels is measured.

The power balance of the system when input power is driven into the outer panels can be calculated by means of the SEA loss matrix, Eq. (22), or the reduced SEA loss matrix, Eq. (23). As only the two power balances corresponding to the excitation on the outer panels are known, the first method based on the cost function $J_{1}$, Eq. (13), is used to determine the effective loss factors of order (2) of the system.

This method can be used straightforwardly and additional constraints may be included in $J_{1}$ from any known characteristic of the system. For the proposed case, whose panels are identical and so are the air layers, the reduced SEA loss matrix of order (2) is symmetric as Eq. (23) shows. Then, the following constraints on the effective loss factors can be imposed:

$$
\begin{aligned}
& \eta_{12}^{(2)}=\eta_{21}^{(2)} \\
& \eta_{23}^{(2)}=\eta_{32}^{(2)} \\
& \eta_{13}^{(2)}=\eta_{31}^{(2)}
\end{aligned}
$$

The effective loss factors of order (2) of this system are shown in Fig. 2 as determined by means of $J_{1}$ taking (or not) into account these additional constraints on the system characteristics. The results are shown along with the actual value of the effective loss factors.

Results show that the EILF of the excited subsystems $\left(\eta_{11}^{(2)}\right.$ and $\left.\eta_{33}^{(2)}\right)$ can be obtained adequately (Fig. 2a) as all the methods agree with the reference values. On the other hand, a large bias is found for the EILF of the subsystems that are not excited $\left(\eta_{22}^{(2)}\right)$ as it is shown in Fig. 2b. On the coupling loss factors, the same influence of the input power is found so that better results are obtained for the ECLF values going out from the excited subsystems (Fig. 2c) than for the ECLF going out from the not excited ones (Fig. 2d). In all the cases the results obtained are closer to the reference when the restrictions in Eq. (24) are used. Finally, regarding the ECLF between the outer panels, Fig. 2e, a large bias is also found. As Eq. (23) shows, this ECLF is related to the resonant transmission through the air layers and the non-resonant transmission through the inner panel, that is not excited in this analysis case. Therefore the same trend identified on the ECLFs associated to the not excited subsystems is expected for this one.

The difference between the loss factors obtained by means of the minimising error method (taking or not the additional constraints) can be evaluated in terms of the energy of the subsystems for a given input power: for instance a unitary power input in all the subsystems. This assessment evaluates the influence on the response prediction and can be calculated solving the SEA equation, Eq. (8), for the actual values of the loss factors, and the results of each method. The energy of each subsystem so calculated is shown in Fig. 3. Energies predicted by application of each method are shown in terms of energy levels references to the energy calculated with the actual values of the loss factors. These results show more clearly the difference between excited and not excited subsystems. Fig. 3a shows that the response of the excited subsystems can be obtained accurately while a noticeable error is expected for not excited ones. The use of additional constraints reduces this error although not in all the frequency range analysed, as shown in Fig. 3b. The improvement of the results due to the additional information is also found for the excited subsystems reducing even further the error in the subsystems response prediction.

\subsection{Partial measurement}

The possibility of using these methods to determine the reduced SEA loss matrix of lower order from the response of a given system known in a higher reduction order is studied in this section. This case corresponds to not measuring (because of impossibility or choice) all the primary subsystems of main interest, $n_{m}<n_{a}$. In this situation the reduced system is modelled by a reduced SEA loss matrix of order $\left(n_{o}\right)$ and size $n_{a} \times n_{a}$ while the power balance measured corresponds to a reduced SEA loss matrix of higher order (lower size $n_{m} \times n_{m}$ ). 


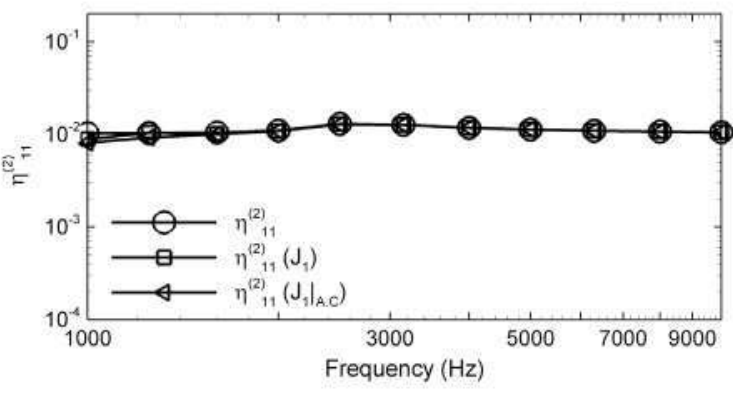

(a)

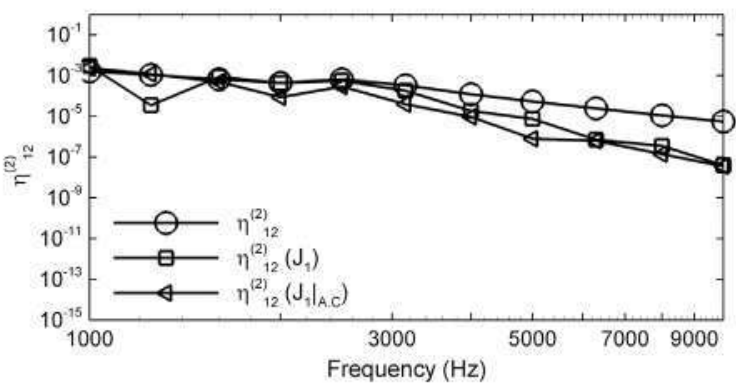

(c)

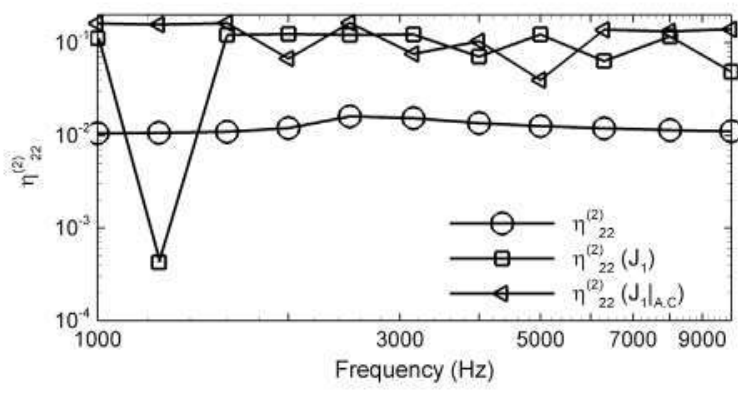

(b)

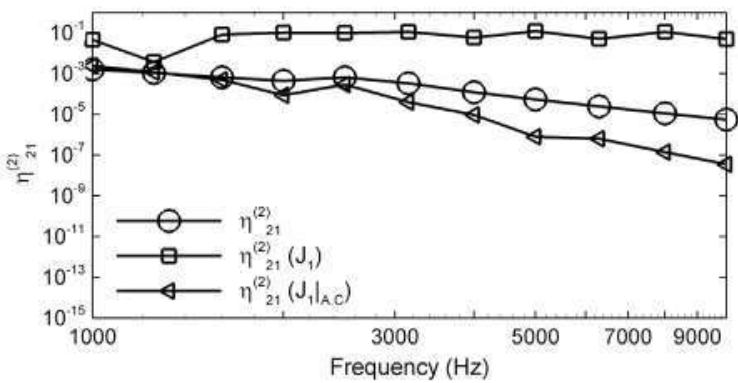

(d)

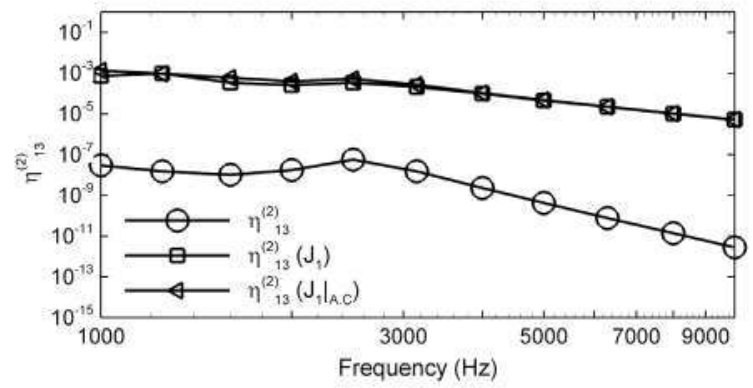

(e)

Fig. 2. Effective loss factors of the validation case determined minimising the functional $\left(f_{1}\right)$ with and without including the additional constraints $($ A.C.) in Eq. (24): (a) ElLF of the outer panels (excited); (b) ElLF of the inner panel (not excited); (c) ECLF from the outer (excited) to inner (not excited) panels; (d) ECLF from the inner (not excited) to the outer (excited) panels and (e) ECLF between outer panels (excited).

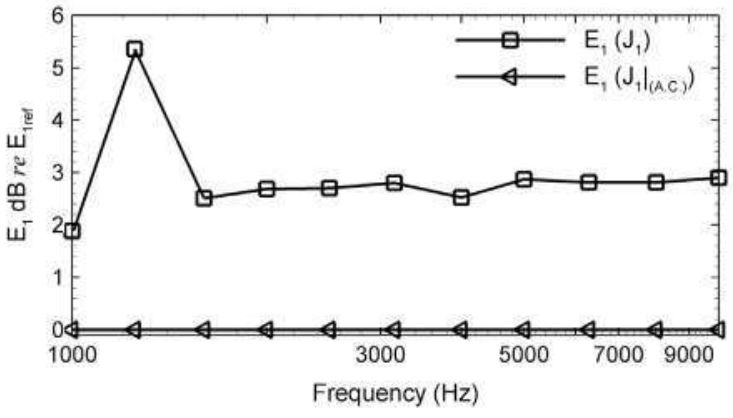

(a)

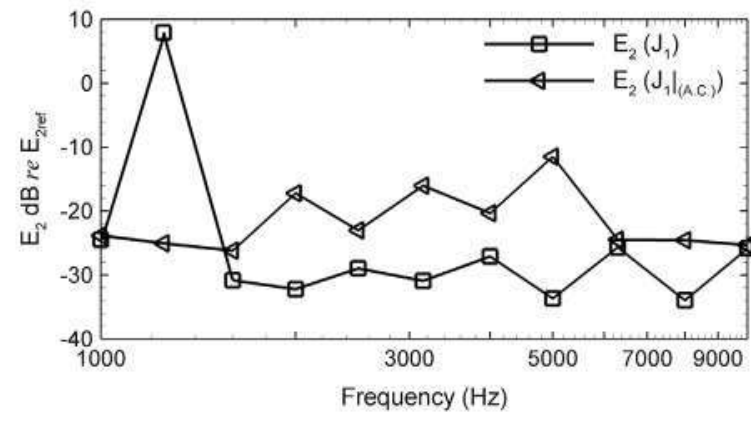

(b)

Fig. 3. Energy levels of the three-subsystem model validation case calculated with each method (minimising the functional $f_{1}$ with and without including the additional constraints) for a unitary power input in all the subsystems. Energy obtained by the actual loss factors is used as reference. (a) Energy of the outer panels and (b) energy of the inner panel. 
The complete system composed of the three panels and the two air layers is considered in order to determine the SEA loss matrix of the system $\mathbf{L}$ of size $5 \times 5$. The two proposed methods (based on functionals $J_{1}$ and $J_{2}$ ) will be applied to different cases. As in Section 4.1, the a priori knowledge of the system characteristics allows defining a set of additional constraints on the loss factors solution based for instance on the identical properties of the panels as can be seen in the SEA loss matrix, Eq. (22)

$$
\begin{aligned}
& n_{12}=n_{54}=n_{32}=n_{34} \\
& n_{13}=n_{31} \\
& n_{35}=n_{53} \\
& n_{21}=n_{23}=n_{43}=n_{45} \\
& n_{14}=n_{15}=n_{25}=n_{41}=n_{51}=n_{52}=0
\end{aligned}
$$

that will be used in combination with functional $J_{1}$.

For the validation of the method based on $J_{2}$ and model updating techniques, a pre-existing SEA model to be updated $\mathbf{L}_{M}$ is needed. This model is defined by including a random error (according to a Gaussian distribution with a relative standard deviation of $20 \%$ ) in the reference values of the SEA loss factors.

Figs. $4 \mathrm{a}-\mathrm{c}$ show that using a pre-existing model accurate results can be obtained while the determination by $J_{1}$ minimisation may produce greater errors in some bands. This conclusion was to be expected as the rank of the solution space is higher. Figs. $4 \mathrm{~d}$ and e show the ILF of the air layers, subsystems that are neither excited nor measured. For this type of subsystems the methods proposed allow estimating the ILF. Regarding the first method, the absence of measured data on the power transmission from these subsystems leads to a higher variability in order to ensure the fulfillment of the additional constraints imposed. The second method, based on updating a previous model, improves the results in general although it depends on the availability of such model. Figs, $4 \mathrm{f}-\mathrm{h}$ show the CLF determined. Results show that the methods proposed provide a good estimation although the solution rank seems to influence the accuracy. Best results are found for the coupling loss factors related to the power transfer from the subsystems analysed. Fig. 4 h shows the results of the direct interaction between the outer panels. The actual value of this CLF is zero as no direct interaction between them exists. Results by means of both methods (minimising $J_{1}$ or $J_{2}$ ) show a level of coupling three orders of magnitude lower than the rest of the CLF, which can be improved imposing the additional constraints.

Results in terms of the subsystems energies show similar findings that for the case of partial excitation. Fig. 5 shows the energy levels of each subsystem calculated with each method for a unitary power input referenced to the energy calculated with the actual loss factors. Again, results can be classified depending on the type of subsystems during the determination of the loss factors with the methods proposed. Energies of subsystems that where considered to be excited (the panels), Figs. 5a-c, show good agreement with respect to the energy of the subsystem calculated with the actual values of the system loss factors. The results regarding the not excited subsystems (air layers), Figs. $5 \mathrm{~d}$ and e, show a lower agreement that can be improved by the application of the method based on model updating.

\section{Application case}

The applicability of the reduced SEA models and the ESEA methods presented is analysed in this section for an actual spacecraft structure with accessibility restrictions: a solar wing in folded configuration. The structure considered is the ARA Mark 3 solar wing developed and manufactured by Dutch Space that was studied within the ESA project Vibro-Acoustics of Large Deployable Structures (VALDS) developed as a cooperation of Dutch Space BV, TAS-Italy, 01 dB-Metravib and the Technical University of Madrid.

The ARA Mark 3 is a three-panel solar wing that in folded configuration are separated by two air layers of $11 \mathrm{~mm}$ thickness. The $2.25 \times 2.75 \mathrm{~m}$ solar panels are $22 \mathrm{~mm}$ thick and they are made up of an aluminium honeycomb core and CFRP skins sandwich structure. The solar panels are fixed by the Hold Down and Release System (HDRS) comprising a set of six structural bushes and four hinges for the wing deployment as shown in Fig. 6 . The structure also involves additional elements as the solar cells or the system wiring as it can be seen in Fig. 7.

Within the VALDS project the Power Injection Method was applied to the solar wing considering the three solar panels as subsystems. A successive excitation on three locations of each panel through an electromagnetic shaker was considered to simulate a non-contact excitation. The vibrational energy of each panel was measured through a set of accelerometers fixed in each panel (ranging from 15 to 21 accelerometers in each panel) [24,25].

Applying PIM to the ARA Mark 3 is conditioned, as for other structures stated above, by the accessibility to the middle panel. In order to apply PIM, excitation on the middle panel had to be driven in the border of the panel uniquely, as it is depicted in Fig. 7. This leads to a different input power spectrum pattern in the middle panel as it is shown in Fig. 8. As Fig. 8 shows this affects specifically to the higher frequency range, where a difference of two orders of magnitude is found.

The power balance of the system, measured through the vibrational energy of the three panels, is shown in Fig. 9 for each power input. 


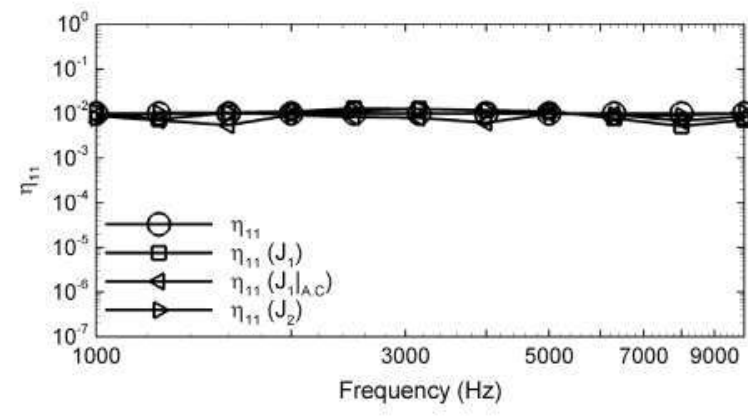

(a)

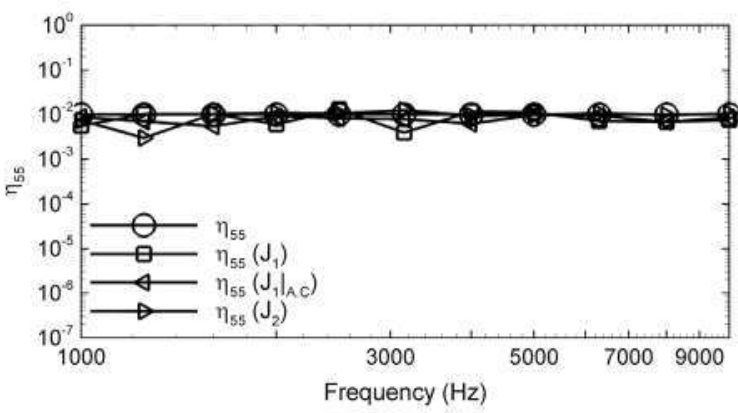

(c)

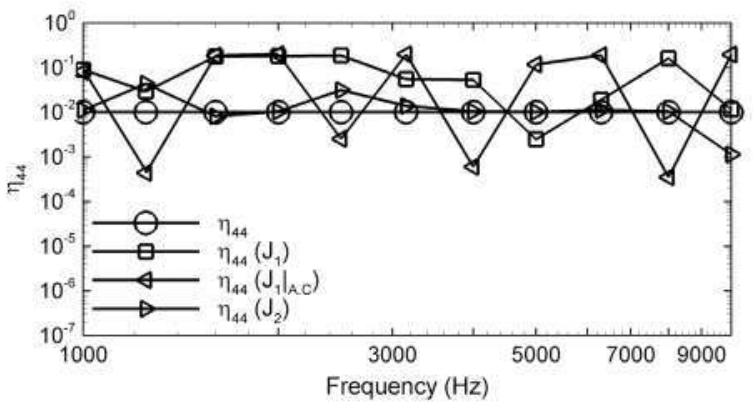

(e)

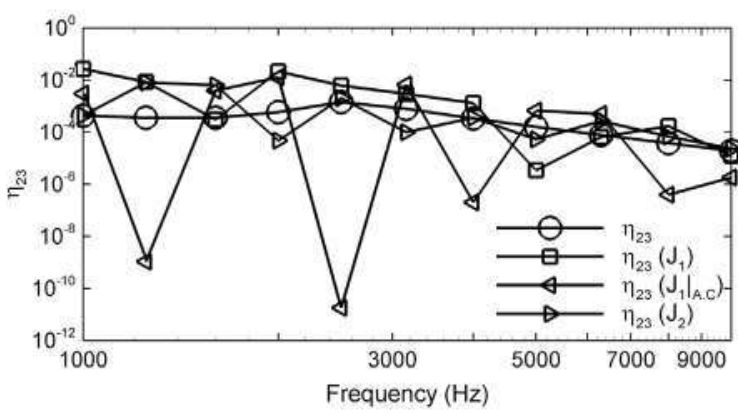

(g)

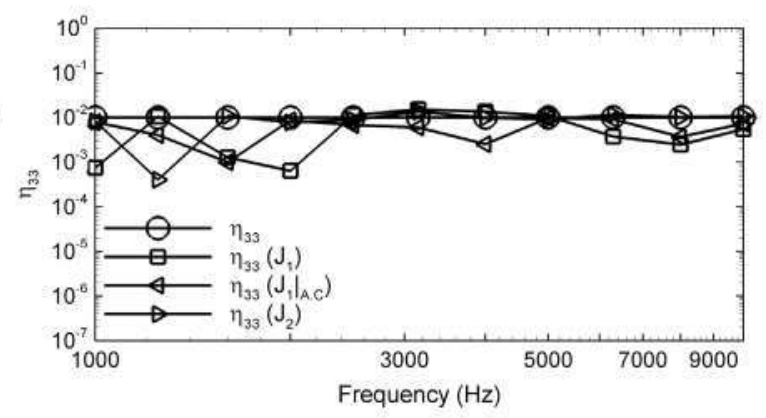

(b)

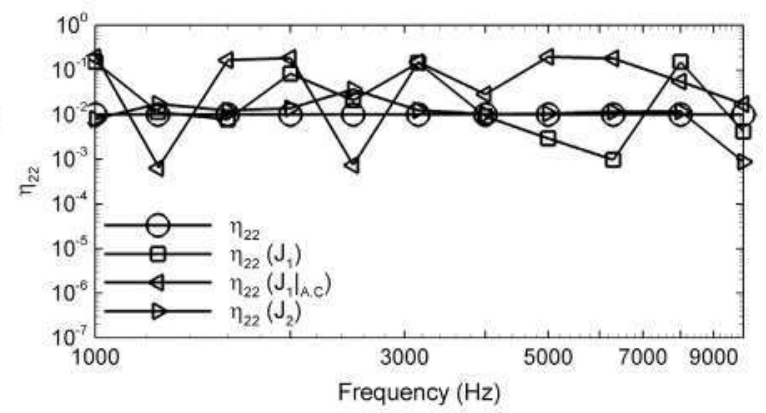

(d)

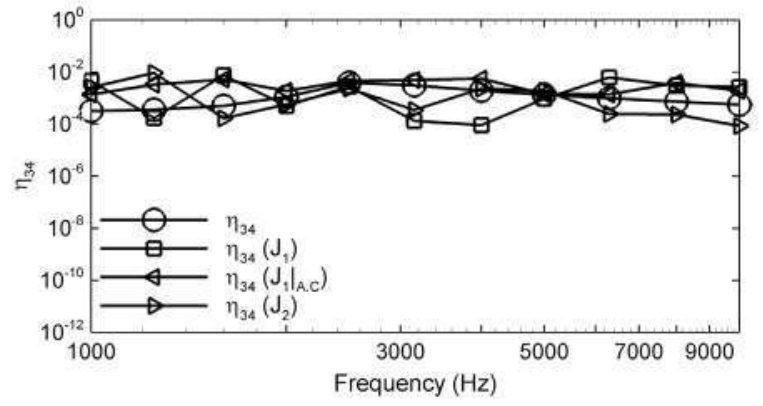

(f)

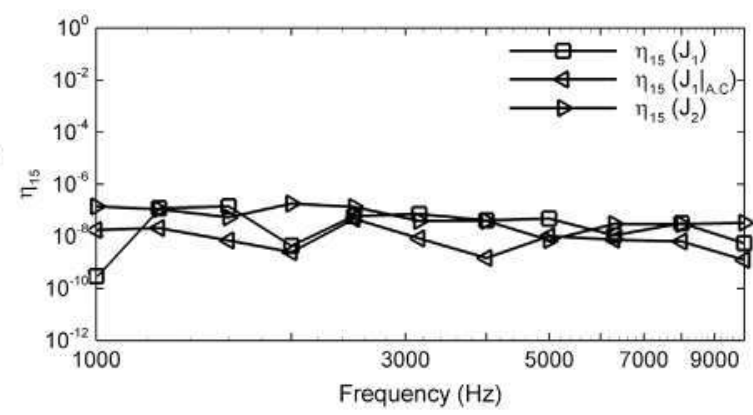

(h)

Fig. 4. SEA loss factors of the validation case determined minimising the functional $J_{1}$ with and without including the additional constraints (A.C.) in Eq. (25), and updating a previous model $\mathbf{L}_{M}$ from the power balance known for three excitation states on the panels: (a) ILF of the lower outer panel (excited); (b) ILF of the inner panel (excited); (c) ILF of upper outer panel (excited); (d) ILF of the lower air layer (not excited); (e) ILF of the upper lower air layer (not excited); (f) CLF from inner panel (excited) to upper air layer (not excited); (g) CLF from lower air layer (not excited) to inner panel (excited); and (h) CLF from lower outer panel (excited) to upper outer panel (excited). 


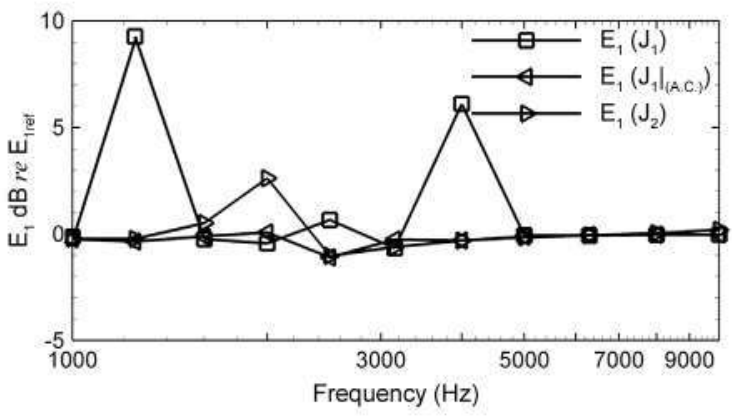

(a)

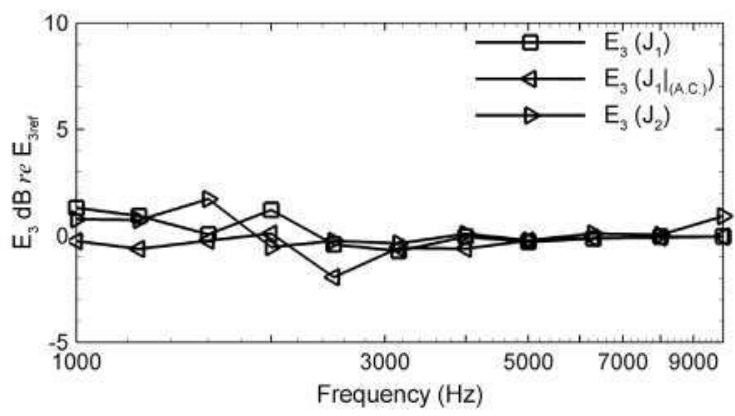

(c)

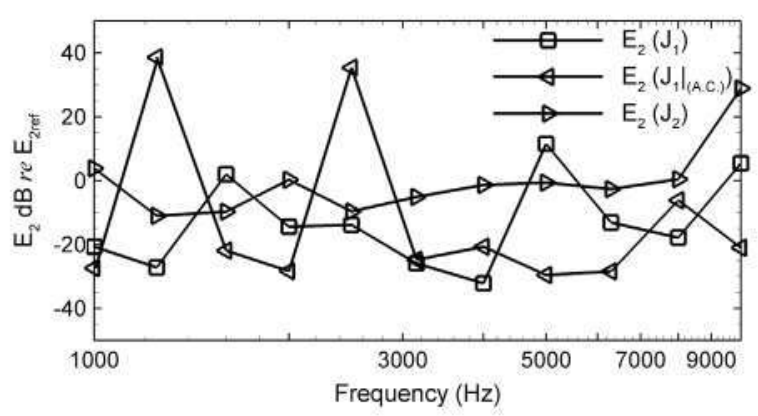

(b)

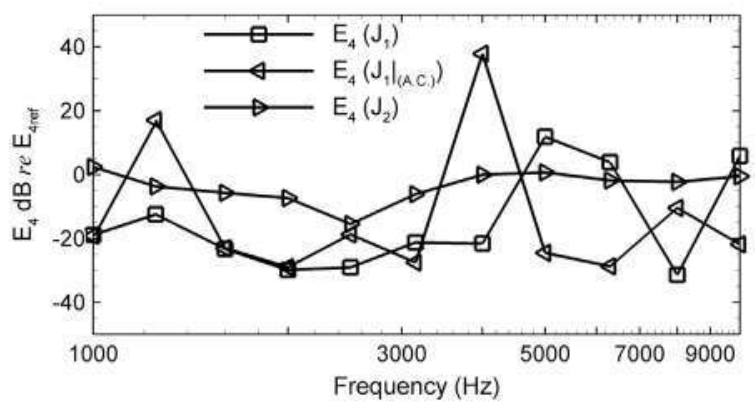

(d)

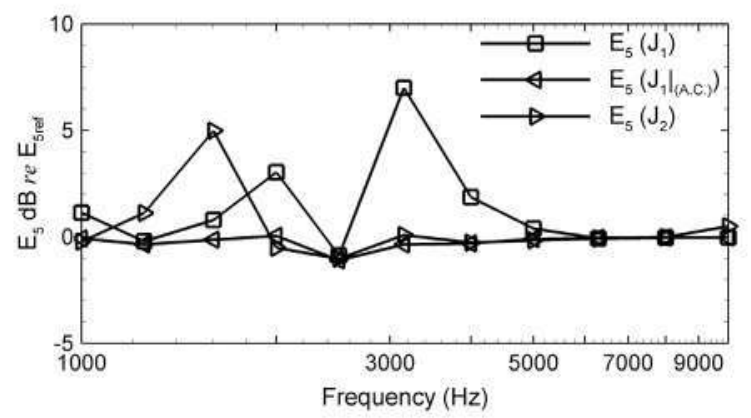

(e)

Fig. 5. Energy levels of the five-subsystem model validation case calculated minimising the functional $J_{1}$ with and without including the additional constraints and minimising $J_{2}$ for a unitary power input in all the subsystems. Energy obtained by the actual loss factors is used as reference. (a) Energy of the lower outer panel (excited); (b) energy of the inner panel (excited); (c) energy of the upper outer panel (excited); (d) energy of the lower air layer (not excited); and (e) energy of the upper air layer (not excited).

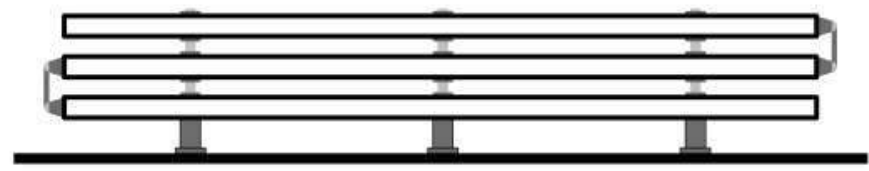

Fig. 6. Sketch of the three-panel solar wing ARA Mark 3 depicting solar panels and HDRS elements.

\subsection{Partial excitation}

If the three solar panels of the ARA Mark 3 are considered as the primary subsystems $\left(n_{a}=3\right)$ and the whole set of power inputs and corresponding energies are considered $\left(n_{e}=n_{m}=3\right)$ then PIM can be used to define the effective loss factors of the system of order 3 , corresponding to reduce out the air surrounding the solar wing and both air layers. The structural excitation of the middle panel gives rise to practical issues related to the fact that the power has to be driven into the plate through its edge. This is why the case of partial excitation (only external panels driven) is considered and the cost function $J_{1}$ 


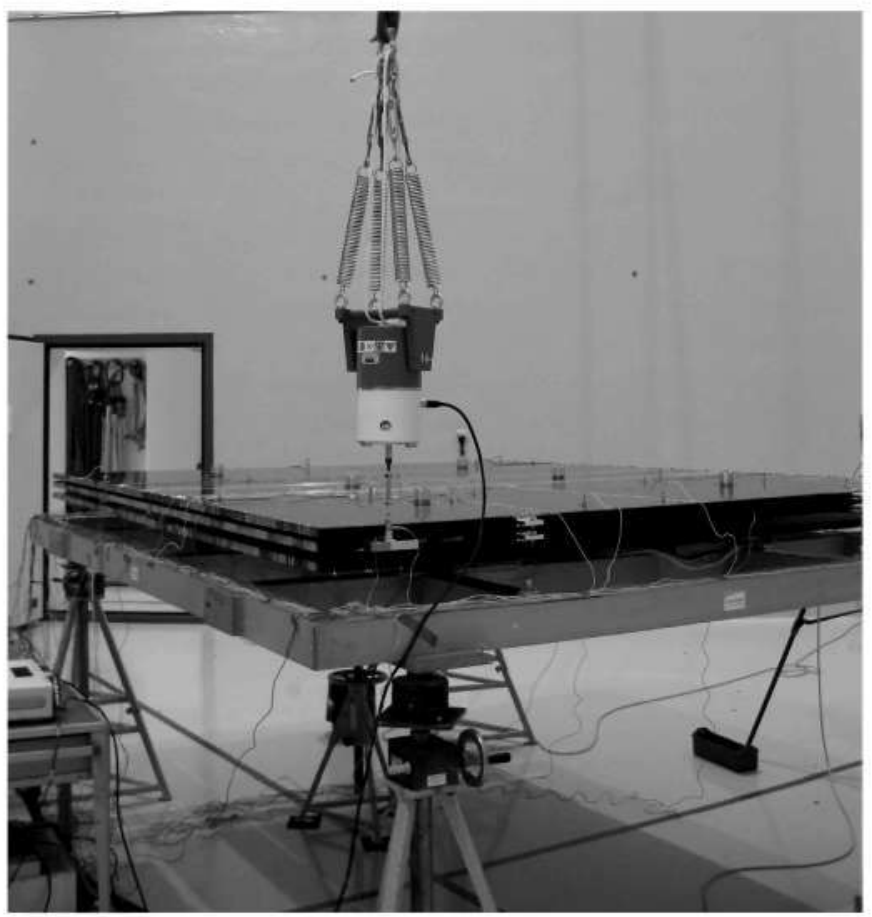

Fig. 7. Photography of the ARA Mark 3 during the Power Injection Method test.

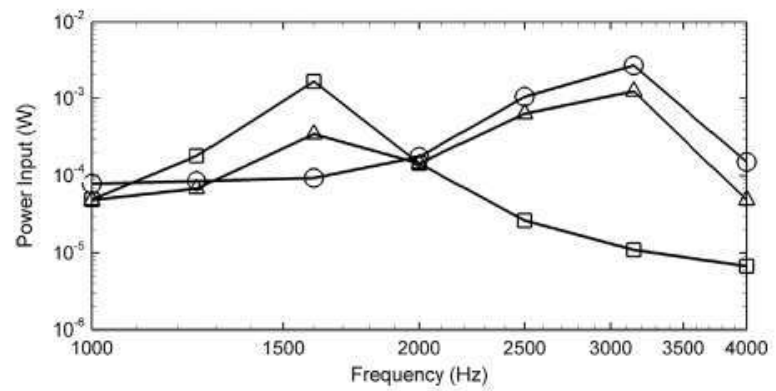

Fig. 8. Input power into the ARA Mark 3 during the PIM test. (- - ) lower panel; (- $\square$-) middle panel; and (- - ) upper panel.

is used to calculate the effective loss factors from the power balances assuming that only the outer panels are excited. These results will be compared with the ones obtained applying PIM to the whole set of measurements.

The effective loss factors of order 3 calculated both through PIM (considering 3 power inputs) and $J_{1}$ minimisation (considering only 2 power inputs) are shown in Fig. 10.

The results can be grouped in two different sets: the excited and the not excited subsystems. On the excited subsystems, Figs. 10a and $\mathrm{c}$ show that the EILF can be obtained accurately by means of the proposed method although only two power inputs are taken into account. Regarding the ECLF between the outer (excited) panels, subsystems 1 and 3, Figs. 10d and e, show a good agreement (though overestimated). About the not excited subsystems a better result is found for the ECLF from excited to not excited subsystems (Figs. $10 \mathrm{f}$ and g) than for the opposite ones (Figs. 10h and i). Even so, these latter ECLF values may be obtained by means of the reciprocity relationship if the modal density of the subsystems involved is known. Finally, as Fig. 10b shows, the EILF of subsystem 2 disagree from the reference values, a further investigation should be carried out to find out if this is a limitation of the method or is related to the numerical problems involved in its resolution.

As shown for the previous cases presented, the energy of the ARA Mark 3 panels is shown in Fig. 11 in terms of energy levels calculated with the loss factors determined by means of the error minimising method referenced to the energy calculated with the loss factors obtained with the application of PIM. Results lead to similar conclusions to the ones from previous cases: the determination of the loss factors with the minimising method from only two input powers provides very low disagreement with PIM results for the excited subsystems (Figs. 11a and c). The energy of the not excited subsystem (inner solar panel) shown in Fig. 11b shows the largest disagreement as expected from previous cases studied. 


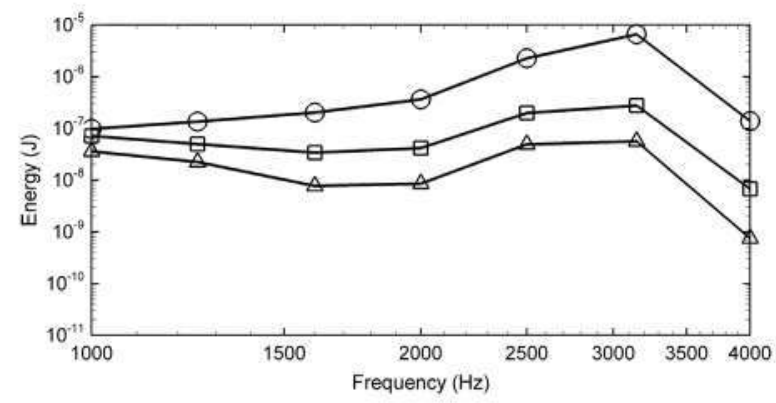

(a)

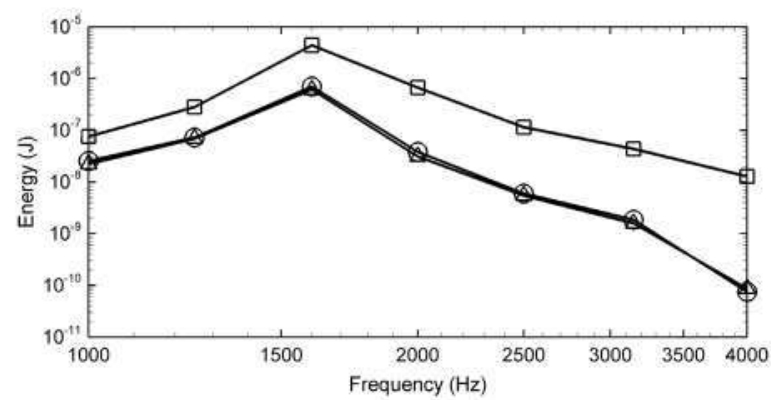

(b)

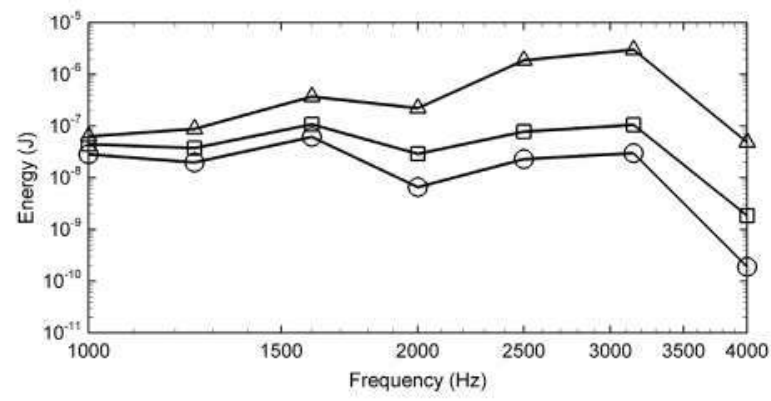

(c)

Fig. 9. Vibrational energy of the (- $\left.\bigcirc_{-}\right)$lower panel, (- - -) middle panel and (- $\left.-\rightarrow\right)$ upper panel for excitation on (a) the lower panel, (b) the middle panel (c) the upper panel.

\section{Conclusions}

Although SEA leads to simple mathematical models from the formal point of view, in practice it may present problems that make it somewhat cumbersome. One of these issues is the difficulty to obtain the loss factors experimentally, especially the CLF. An ESEA requires that input power is injected (and measured) sequentially in all the subsystems, what leads to practical problems related with accessibility and time (large systems). A method to overcome this problem is to split the whole system into groups of two or three coupled subsystems, this way a hard problem is substituted by a set of easier ones. This method is equivalent to what in this work has been called truncation, and it has proven to give good results in areas such as building acoustics. In this paper an alternative method is suggested based on matrix reduction techniques. It has the advantage that it maintains the information on the energy propagating through the omitted subsystems. Even more, the reduced model is able to estimate the energy (or effective loss factors) of not measured primary subsystems, something impossible with a truncated model. A consequence is that these reduced models can replace the complete models in the analysis, provided that they include all the subsystems of interest. As the effective coefficients include the information of the complete system the results are related with it and not with the truncated system. On the other hand, as effective loss factors of order $(k)$ are related to actual loss factors, reduced models can be used to estimate the later in structures where the ESEA cannot be fully applied using numerical techniques or inverse methods.

The reduced models have been applied to a solar wing in folded configuration. In this case, input power cannot be injected into the air layers, so that a standard ESEA could not be used (apart from the fact that the need to drive power into the middle panel makes the experimental set up more complicated). Results show that the ILF of the source subsystems and 


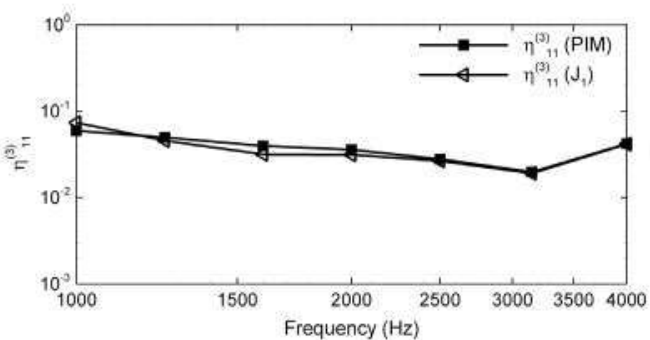

(a)

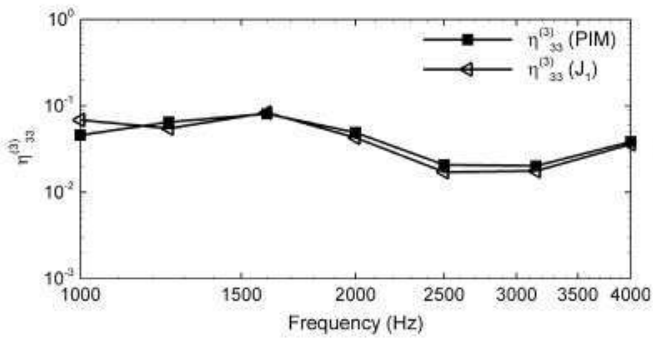

(c)

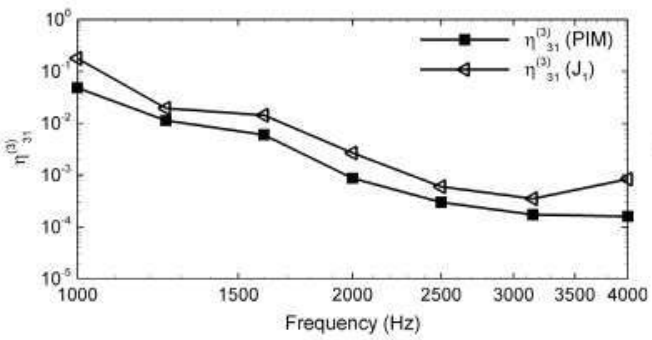

(e)

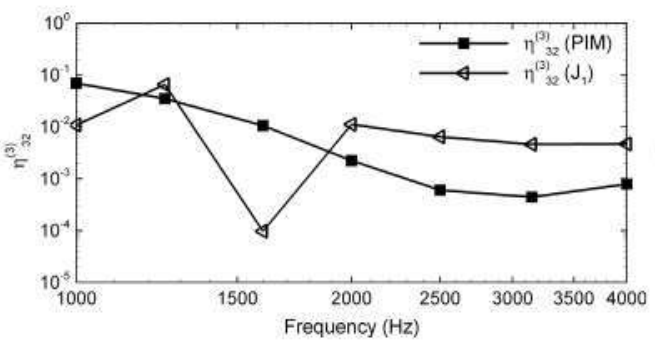

(g)

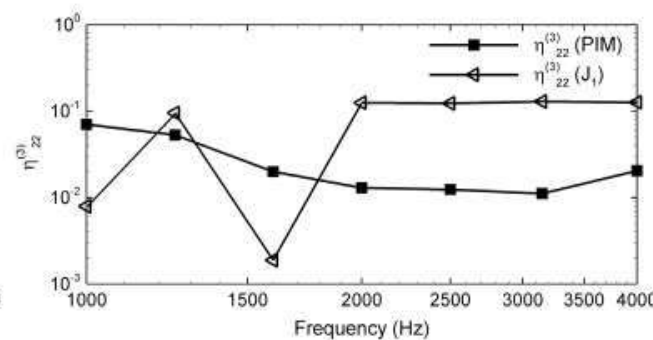

(b)

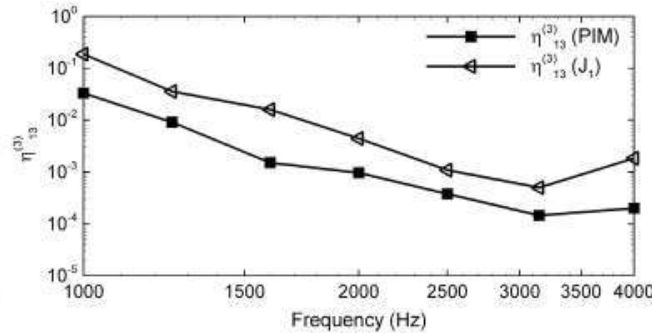

(d)

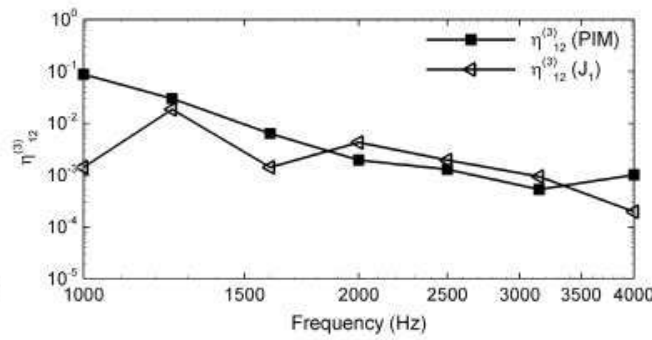

(f)

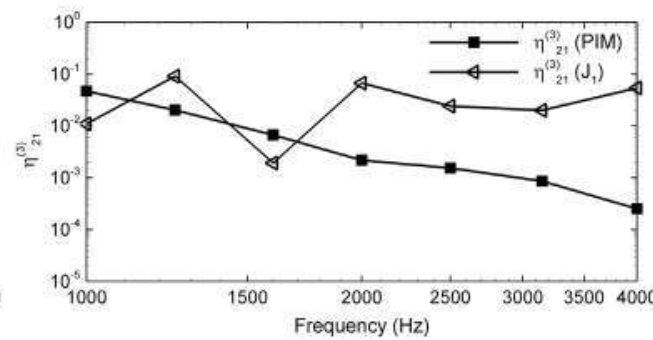

(h)

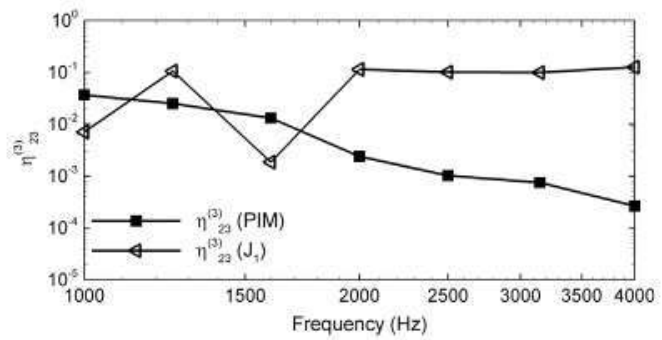

(i)

Fig. 10. Effective loss factors of order 3 of the ARA Mark 3 calculated by PIM and $J_{1}$ minimisation: (a) ElLF of the lower outer panel; (b) EILF of the inner panel; (c) EILF of upper outer panel; (d) ECLF from lower outer panel to the upper outer one; (e) ECLF from upper outer panel to the lower outer one; (f) ECLF from lower outer panel to the inner one; ( $g$ ) ECLF from upper outer panel to the inner one; $(h)$ ECLF from the inner panel to the lower outer one; and (i) ECLF from the inner panel to the upper outer one. 


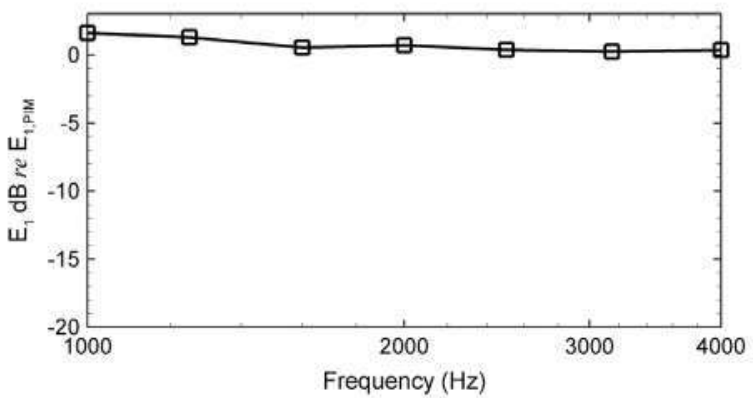

(a)

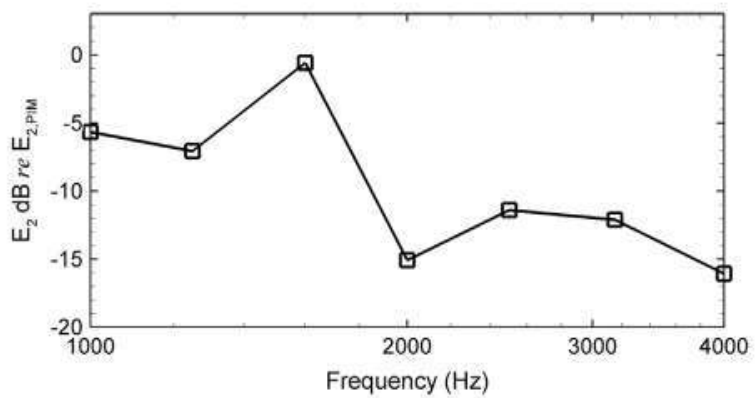

(b)

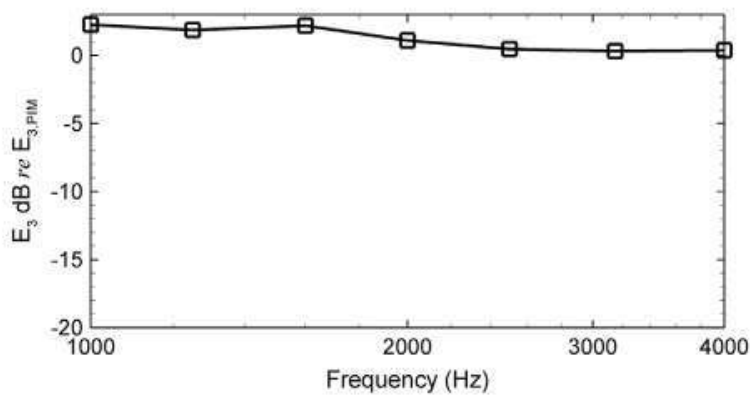

(c)

Fig. 11. Energy levels of the ARA Mark 3 calculated minimising $J_{1}$ for a unitary power input. Energy calculated by the loss factors obtained applying PIM are used as reference. (a) Energy of the lower outer panel; (b) energy of the inner panel (not excited); and (c) energy of the upper outer panel (excited).

the CLF going out from the source subsystems are correctly determined. However, the method fails to estimate the loss factors of the omitted subsystems (despite, these CLF can be obtained by reciprocity). Despite this fact, the analysis of the energy of the system corresponding to the set of effective loss factors determined through each method shows that the energy of the excited subsystems can be correctly predicted while the energy of the not excited subsystems shows a much lower agreement with the reference response (the actual one for the validation case and the PIM result for the application case). This result is expected due to the lower knowledge on the not excited subsystems as no information on the power transfer from them is known. Nevertheless, the variability of the results on different frequency bands suggests that numerical implications of the methods implementation should be further investigated. From a general point of view, the error minimisation method taking into account additional constraints provides the best results if no previous model is available. Nevertheless, this method could be used to define the previous model so that the model updating method could be used. It is expected that this nested procedure should include the advantages of both methods.

\section{Acknowledgements}

M. Chimeno Manguán acknowledges the mobility grant from the Consejo Social of the Technical University of Madrid within the 11th Internationalization Fellowships Program.

\section{References}

[1] R.H. Lyon, R.G. Dejong, Theory and Applications of Statistical Energy Analysis, 2nd ed., Butterworth-Heinemann, Newton, Ed., 1995.

[2] L. Maxit, J.L. Guyader, Estimation of SEA coupling loss factors using a dual formulation and FEM modal information. Part I; theory, J. Sound Vib. 239 (5) (2001) 907-930.

[3] D. Bies, S. Hamid, In situ determination of loss and coupling loss factor by the power injection method, J. Sound Vib. 70 (2) (1980) $187-204$.

[4] N. Lalor, Practical Considerations for the Measurement of Internal and Coupling Loss Factors on Complex Structures, Technical Report ISVR no. 182, 1990.

[5] C. Hopkins, Experimental statistical energy analysis of coupled plates with wave conversion at the junction, J. Sound Vib. 322 (2009) $155-166$.

[6] B. Cimerman, T. Bharj, G. Borello, Overview of the experimental approach to statistical energy analysis, in: Proceedings of the SAE Noise and Vibration Conference, Paper 97NV169, 1997, pp. 1-6.

[7] K. Renji, M. Mahalakshmi, High frequency vibration energy transfer in a system of three plates connected at discrete points using statistical energy analysis, J. Sound Vib. 296 (3) (2006) 539-553.

[8] F. Fahy, An alternative to the sea coupling loss factor: rationale and method for experimental determination, J. Sound Vib. 214 (2) (1998) $261-267$.

[9] O. Guasch, A direct transmissibility formulation for experimental statistical energy analysis with no input power measurements, $J$. Sound Vib. 330 (25) (2011) 6223-6236. 
[10] C. Hopkins, Statistical energy analysis of coupled plate systems with low modal density and low modal overlap, J. Sound Vib. 251 (2) (2002) 193-214.

[11] C.C. Flanigan, Model reduction using Guyan, IRS, and dynamic method, in: Proceedings of the 16th International Modal Analysis Conference, 1998.

[12] R.]. Guyan, Reduction of stiffness and mass matrices, AlAA J. 3 (2) (1965) 30.

[13] J.E. Mottershead, M.I. Friswell, Model updating in structural dynamics: a survey, J. Sound Vib. 167 (2) (1993) 347-375.

[14] A. Nobari, M. Imregun, S. Ziaei Rad, On the uniqueness of updated model, in: Proceedings of the 19th International Seminar on Modal Analysis, Leuven, Belgium, 1994, pp. 151-163.

[15] M.I. Friswell, J.E. Mottershead, Finite Element Model Updating in Structural Dynamics, Springer, Netherlands, 1995.

[16] Z. Ugray, L. Lasdon, J. Plummer, F. Glover, J. Kelly, R. Marti, Scatter search and local NLP solvers: a multistart framework for global optimization, INFORMS J. Comput. 19 (3) (2007) 328-340.

[17] F. Glover, A template for scatter search and path relinking, in: Lecture Notes on Computer Science, vol. 1363, 1998, pp. 1-51.

[18] R.J.M. Craik, R.S. Smith, Sound transmission through double leaf lightweight partitions. Part I: airborne sound, Appl. Acoust. 61 (2) (2000) 223-245.

[19] R.J.M. Graik, R. Wilson, Sound transmission through masonry cavity walls, J. Sound Vib. 179 (1) (1995) 79-96.

[20] F.D. Hart, K.C. Shah, Compendium of modal densities for structures, Teclnnical Report NASA No. C-1773, 1971.

21] C.W. de Silva, Vibration, damping, control and design, in: Mechanical and Aerospace Engineering Series, Taylor \& Francis, Bosa Roca, 2007.

[22] F.G. Leppington, E.G. Broadbent, K.H. Heron, Susan M. Mead, Resonant and non-resonant acoustic properties of elastic panels. I. The radiation problem, Proc. R. Soc. Lond. 406(August (1831)) (1986) 139-171.

[23] D. Palumbo, Estimating sound power radiated from rectangular baffled panels using a radiation factor, J. Acoust. Soc. Am. 126 (4) (2009) 1827-1837.

[24] 01 dB-Metravib, Breadboard test Instrumentation (AF000375/RAP/004/A), in: Vibro-Acoustic Analysis and Test Methods for Large Deployable Structures (VAA-TM-LDS) DS-VAN2-09/003-NL/TS, 2009.

[25] 01 dB-Metravib, Breadboard Test Results (AF000375/RAP/006/A), in: Vibro-Acoustic Analysis and Test Methods for Large Deployable Structures (VAATM-LDS) DS-VAN2-09/004-NL/TS, 2010. 